ЧЕРНИКОВА О.П., ЗЛАТИЦКАЯ Ю.А., КЛИМАШИНА Ю.С.

УдК 338.2

ЧЕРНИКОВА ОКСАНА ПЕТРОВНА

к.э.н., доцент, Сибирский государственный индустриальный университет, заведующая кафедрой экономики, учета и финансовых рынков. АБ «Кузнецкбизнесбанк», e-mail: chernikovaoo@yandex.ru

ЗЛАТИЦКАЯ ЮЛИЯ АЛЕКСАНДРОВНА к.т.н., доиент, Сибирский государственный индустриальный университет, учета и финансовых рынков. АБ «Кузнеикбизнесбанк», e-mail: chernikovaop@yandex.ru

КЛИМАШИНА ЮЛИЯ СЕРГЕЕВНА к.э.н., доцент, Сибирский государственный индустриальный университет, учета и финансовых рынков. АБ «Кузнецкбизнесбанк», e-mail: chernikovao $@$ @yandex.ru

\title{
МЕХАНИЗМ ЭКОНОМИЧЕСКОЙ ОЦЕНКИ РЕЦЕПЦИИ «ЗЕАЕНОЙ॥ ЭНЕРГЕТИЧЕСКОЙ ПРАКТИКИ ПОГРАНИЧНЫХ РЕГИОНОВ
}

Аннотадия. Цель работы. Целью работы является исследование процесса изменения энергетической парадигмы в отечественной и мировой экономике, анализ возможностей рецепиии «зеленой» энергетической практики пограничных регионов, разработка механизма экономической оценки ее результатов и схемы его интеграции в плановоэкономическую деятельность предприятия. Метод или методология проведения работы. Исследование основывается на общенаучной методологии с использованием методов: научной абстракиии, диалектического развития, абстрактной логики, моделирования учетных проиессов, контент-анализа, обобщения и др. Основой работы являются научные публикации отечественных и зарубежных ученых, раскрывающие проблематику внедрения в хозяйственную практику предприятий и регионов альтернативных энергетических технологий. Результаты. B работе установлено, что в условиях динамично развивающейся и непрерывно трансформирующейся рыночной экономики, неразрывно связанной с проиессом глобализации, сырьевая модель экономического роста становится неэффективной в долгосрочной перспективе, вследствие чего происходят структурные изменения использования энергетической практики пограничных регионов, связанные с поиском новых форм организации пространства и повышения конкурентоспособности территории, позволяющие более эффективно использовать потенциал регионов. Одним из основных стратегических направлений развития мировой энергетики предусматривается обеспечение сущиественного снижения техногенной нагрузки на окружающую среду с ее сохранением в благоприятном для жизнедеятельности человека состоянии посредством внедрения «зеленых» энергетических практик в деятельность каждого конкретного предприятия. Использование данных практик должно обеспечить переход от ископаемых топлив к возобновляемым источникам энергии, рост энергоэффективности экономики.

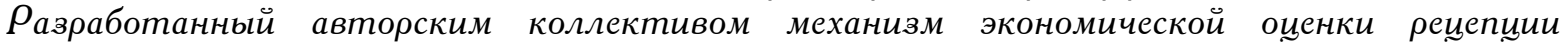
«зеленой» энергетической практики пограничных регионов предложено интегрировать в планово-экономическую деятельность предприятий. Его использование позволит оптимизировать показатели энергоемкости технологических проиессов, обеспечивающих выполнение плановых показателей деятельности предприятий в области энерго- и экономической эффективности, экологической безопасности территорий, повышения качества жизни населения и сохранения ресурсов для будущихх поколений. Область прнменения

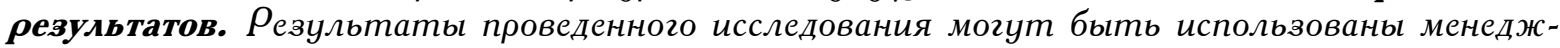
ментом предприятий при планировании показателей производственно-хозяйственной деятельности и принятии управленческих решений, а также региональными органами власти при разработке программ повышения энергоэффективности территорий. Выводы. Исследования и разработки в энергетической отрасли требуют своевременного предвидения социально-экономических изменений и формулирования траектории внедрения «зеленых» энергетических технологий в технологические проиессы предприятия с расче- 
том основных экономических показателей эффективности от таких нововведений в организации.

Ключевые слова: регион, природные ресурсы региона, «зеленая» энергетическая практика, энергоэффективность, альтернативные энергетические технологии, возобновляемые источники энергии.

CHERNIKOVA OKSANA PETROVNA

Ph. D., associate Professor, Siberian state industrial University, head of the Department of Economics, accounting and financial markets. $A B$ "kuznetskbusinessbank», e-mail: chernikovao $@$ @yandex.ru

ZLATITSKAYA YULIYA ALEKSANDROVNA

$\rho$ h. D., associate Professor, Siberian state industrial University, head of the Department of Economics, accounting and financial markets. AB "kuznetskbusinessbank», e-mail: chernikovaop@yandex.ru

KLIMASHINA JULIA SERGEEVNA

Ph. D., associate Professor, Siberian state industrial University, head of the Department of Economics, accounting and financial markets. AB" kuznetskbusinessbank», e-mail: chernikovao $@ y a n d e x . r u$

\title{
MECHANISM OF ECONOMIC EVALUATION OF RECEPTION " GREEN॥ ENERGY PRACTICES OF BORDER REGIONS
}

\begin{abstract}
Purpose of work. The aim of the work is to study the process of changing the energy paradigm in the domestic and world economy, to analyze the possibilities of reception of "green" energy practices of border regions, to develo $a$ mechanism for economic evaluation of its results and the scheme of its integration into the planned economic activity of the enterprise. Method or methodology of the work. The research is based on General scientific methodology using methods: scientific abstraction, dialectical development, abstract logic, modeling of accounting processes, content analysis, generalization, etc. The basis of the work is the scientific publications of domestic and foreign scientists, revealing the problems of introduction of alternative energy technologies into the economic practice of enterprises and regions. Results. In work it is established that in conditions of dynamic and continuously transforming market economy is inextricably linked with the globalization process, the raw model of economic growth becomes ineffective in the long term, as a result of structural changes in the energy use practices of border regions related to the search for new forms of space organization and competitiveness of the territories, allowing more efficient use of the potential of the regions. One of the main strategic directions of the world energy development is to ensure a significant reduction of the technogenic load on the environment with its preservation in a favorable state for human life through the introduction of "green" energy practices in the activities of each individual enterprise. The use of these practices should ensure the transition from fossil fuels to renewable energy sources, the growth of energy efficiency of the economy. The mechanism of economic evaluation of reception of "green" energy practice of border regions developed by the author's team is proposed to be integrated into the planned economic activity of enterprises. Its use will optimize the energy intensity of technological processes, ensuring the achievement of planned targets of activity of the enterprises in the area of energy and economic efficiency, ecological safety of the territories, improve the quality of life and preserving resources for future generations. The scope of the results. The results of the study can be used by the management of enterprises in the planning of indicators of production and economic activity and management decisions, as well as regional authorities in the development of programs to improve energy efficiency of territories. Summary. Research and development in the energy industry requires timely foresight of socio-economic changes and the formulation of the trajectory of the introduction of "green" energy technologies in the technological processes of the enterprise with the calculation of the main economic performance indicators from such innovations in the organization.
\end{abstract}

Keywords: region, natural resources of the region, "green" energy practice, energy efficiency, alternative energy technologies, renewable energy sources. 
Введение. В настоящее время использование «зеленой» энергетической практики в производственно-хозяйственной деятельности предприятий и организаций, находящей отражение в различных отечественных и мировых исследованиях [1-4], предусматривает достижение равновесия экономических, экологических и социальных потребностей общества.

Критический анализ работ, посвященных проблемам перехода к «зеленой» энергетической практике, позволил сделать однозначный вывод о том, что основные направления такого перехода тесно связаны с развитием альтернативных энергетических технологий [5-13].

Основная идея концепции «зеленой» энергетической практики пограничных регионов предусматривает, что в процессах производства энергии замена невозобновляемых природных ресурсов на устойчивые должна сопровождаться повышением качества жизни населения, соблюдением требований по охране окружающей среды, рациональным природопользованием в целях оптимизации жизнедеятельности настоящего и будущих поколений с учетом возможного потенциального взаимодействия регионов.

При этом планирование хозяйственной деятельности с учетом пространственной принадлежности предприятий и организаций должно быть направлено на использование в производстве «зеленых» энергетических практик пограничных регионов, что обеспечит снижение суммарного объема выбросов и сбросов загрязняющих веществ и соответствие требованиям экологической безопасности [5].

Обладая схожими природно-климатическими условиями и ресурсами, пограничные регионы могут осуществлять рецепцию «зеленых» энергетических практик, совместно решить возникающие проблемы энергоснабжения и реализовать инвестиционные проекты, представляющие интерес для обеих сторон.

Методы исследования. Основой данного исследования являются изучение, анализ российского и мирового опыта использования альтернативных видов энергии. Для всестороннего и целостного рассмотрения этой проблемы был использован системный подход. Методологической базой исследования являлась приведенная в списке источников научная и учебная литература.

На первоначальном этапе при постановке цели и формулировке задач авторским коллективом был использован абстрактно-логический метод исследования для изучения возможностей рецепции «зеленых» энергетических практик пограничных регионов.

Для аналитического обзора достоинств и недостатков устойчивых энергетических технологий, выявления их видов, закономерностей развития и причинно-следственных связей применялся метод сравнительного анализа и синтеза.

На этапе разработки механизма планирования различных вариантов устойчивых технологий с учетом рисков перехода/не перехода к их использованию и оценки экономической эффективности рецепции «зеленых» энергетических практик предприятий пограничных регионов применялись методы научной абстракции, диалектического развития, моделирования учетных процессов. Эвристические методы в сочетании с логическими применялись при проведении дискуссии.

В условиях всевозрастающего дефицита природных и финансовых ресурсов «обостряется» проблема их экономии у всех участников рынка, то есть в современных реалиях ресурсосберегающие технологии становятся приоритетными не только для производителей, но и заслуживают особого внимания у потребителей товаров, работ, услуг [1].

Нельзя не отметить и тот факт, что энергосбережение является не только важнейшей внутрироссийской проблемой, но и международной, так как от этого напрямую зависит получение дополнительных топливно-энергетических ресурсов для экспорта, которые в свою очередь повысят стабильность и надежность российских поставок на европейский рынок.

На современном этапе развития общества энергия является ключевым фактором экономического роста и повышения благосостояния, но в связи с постоянно увеличивающимся спросом на нее возникает объективная необходимость в создании прорывных технологий и освоении новых энергоносителей даже в регионах, в полной мере обеспечивающих свои энергетические потребности за счет ископаемого топлива, что, в свою очередь, приводит к смене технологической парадигмы и диверсификации производства в сторону малозатратных, малоотходных и малотоксичных «зелёных технологий». 
В последние годы значение межрегионального и приграничного сотрудничества объективно растет. «Зеленая» энергетическая практика пограничных регионов включает в себя не только производство энергии из возобновляемых источников, таких как гидроэлектроэнергия, ядерная и солнечная энергия, энергия ветра, геотермальная энергия, энергия приливов, биомасса, водород, наряду с повышением эффективности использования и экологическим управлением, но и экономическую оценку результатов такой рецепции.

Ряд стран и регионов успешно решают проблемы энергообеспечения на основе использования в деятельности компаний возобновляемой энергетики, о чем свидетельствует анализ российского и международного опыта применения устойчивых энергетических технологий.

В процессе проведения исследований и написания статьи было изучено большое количество теоретических и практических работ по применению альтернативных энергетических технологий, однако методология исследований экономической оценки заимствования энергетической практики пограничных регионов в каждой из них остается за пределами целостного предмета научного видения, а комплексный подход к экономической оценке такой рецепции в тени научных изысканий [14-22].

Гипотеза настоящего исследования построена на предположении о том, что внедрение и использование в работе компаний механизма экономической оценки рецепции «зеленой» энергетической практики пограничных регионов будет способствовать всестороннему и устойчивому обеспечению стабильного развития предприятия и общества в целом.

Для принятия решения о возможности и целесообразности использования в деятельности компании «зеленой» энергетической практики пограничных регионов необходимо провести комплекс работ, позволяющих оценить экономический и экологический эффекты от внедрения в энергетический баланс. Важная роль здесь отводится именно процессу моделирования и оценки влияния релевантных решений на итоговые показатели финансово-хозяйственной деятельности предприятия.

Результаты. Авторским коллективом был разработан механизм экономической оценки рецепции «зеленой» энергетической практики пограничных регионов, который, по нашему мнению, должен быть интегрирован в планово-экономическую деятельность предприятия (рисунок 1) и реализовываться со второго по четвертый этап.

Этап 1. Планирование финансовых результатов деятельности предприятия на предстоящий период, на основании выполнения которого менеджмент предприятия устанавливает плановые показатели объема производства и себестоимости единицы продукции.

Этап 2. Экономическая оценка рецепции «зеленой» энергетической практики пограничных регионов. Предусматривает: определение энергоемкости технологических процессов предприятия, обеспечивающей выполнение плановых показателей объема производства и себестоимости продукции; мониторинг «зеленых» энергетических практик пограничных регионов и сравнительный анализ их климатических условий с регионом пребывания предприятия для определения возможностей рецепции технологий; оценку рисков перехода (или не перехода) на «зеленые» энергетические практики с позиции реформирования законодательства, необходимых трансформаций в технологических процессах, изменений спроса в зависимости от степени экологичности продукции, преобразования деловой репутации; расчет капитальных затрат и оценку эффективности инвестиционного проекта по внедрению устойчивых энергетических технологий.

Кроме того, на данном этапе должна быть произведена оценка финансового воздействия энергетической рецепции «зеленых» технологий на показатели форм финансовой отчетности предприятия, коэффициенты эффективности использования ресурсов, уровень энергоемкости производства, рост конкурентоспособности продукции, укрепление деловой репутации.

Этап 3. Принятие управленческого решения о рецепции «зеленой» энергетической практики и реализация мероприятий по ее внедрению предполагает финансирование рецепции «зеленых» энергетических технологий, организацию работ по их внедрению в технологические процессы предприятия, обучение работников использованию новой энергетической практики. 
ЧЕРНИКОВА О.П., ЗЛАТИЦКАЯ Ю.А., КЛИМАШИНА Ю.С.

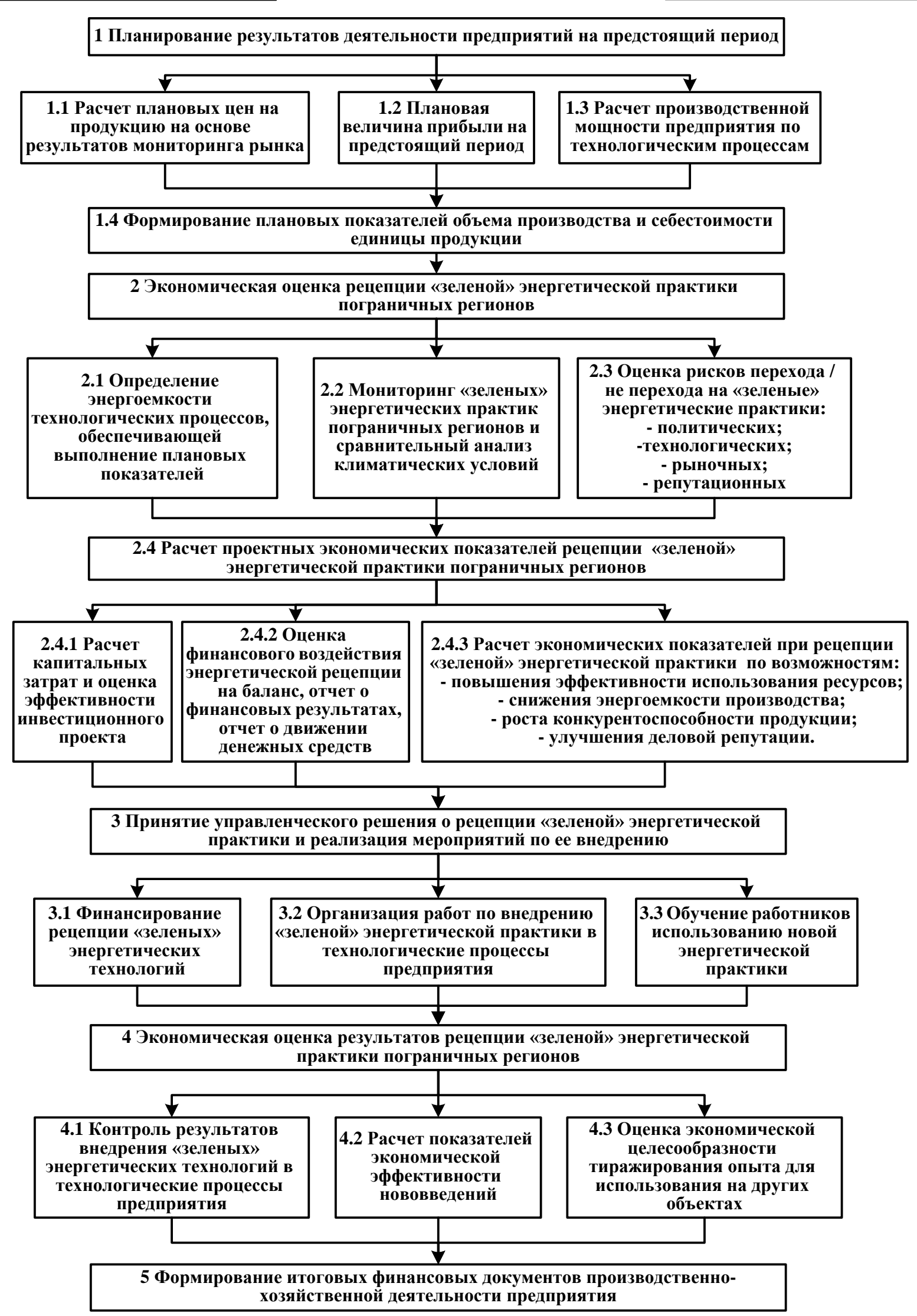

Рис. 1. Интеграция механизма экономической оценки рецепции «зеленой» энергетической практики пограничных регчонов в планово-экономическую деятельность предприятия. 
Этап 4. Экономическая оценка результатов рецепции «зеленой» энергетической практики пограничных регионов предусматривает контроль результатов внедрения мероприятий в технологические процессы предприятия, расчет показателей экономической эффективности нововведений (экономия затрат в сферах теплоснабжения и электроснабжения, прибыль, чистый дисконтированный доход, рентабельность инвестиций, срок окупаемости капитальных вложений) и региона (размер дотаций, субсидий и инвестиций на развитие энергетического потенциала региона, экономия средств бюджетов на тепло- и энергоснабжение), показатели энергоэффективности организации (экономия теплоэнергии, экономия электроэнергии, энергоемкость технологических процессов и в целом деятельности организации, удельный расход электроэнергии на производство единицы продукции) и региона (энергоемкость секторов промышленности, жилищного сектора, энергоемкость валового регионального продукта, интегральный индекс энергоэффективности, снижение объемов выбросов и сбросов в окружающую среду). На основании произведенных расчетов менеджмент предприятия может принять решение о целесообразности или нецелесообразности тиражирования опыта использования «зеленой» энергетической практики на других объектах.

Этап 5. Формирование итоговых финансовых документов производственнохозяйственной деятельности предприятия, в которых отражаются результаты рецепции «зеленой» энергетической практики пограничных регионов.

Выводы. Основным двигателем процесса глобализации, несомненно, является переход мировой экономики к новому технологическому укладу, который предполагает повышение эффективности производства и конкурентоспособности, а также модернизацию технологической базы (с возможным поиском альтернативных производств) для обеспечения улучшения качества жизни и среды обитания.

«Зеленая» энергетическая практика как составной элемент концепции устойчивого развития обладает значительным потенциалом. В рамках рецепции «зеленой» энергетической практики пограничных регионов существует достаточно возможностей для перехода к ресурсоэффективным и энергосберегающим бизнес-моделям. Разработанная авторским коллективом интеграция механизма экономической оценки рецепции «зеленой» энергетической практики пограничных регионов в планово-экономическую деятельность предприятия не предусматривает значительных инвестиций для ее применения на практике, вследствие чего может стать одним из основных направлений прорывного развития производства в ближайшие годы.

Предлагаемый механизм нужно рассматривать как единый процесс, в равной мере охватывающий все стороны деятельности компании, помимо производства и потребления, при этом ведущая роль в решении глобальных задач по устойчивому развитию современного и будущего общества принадлежит менеджменту компании.

Использование разработанного механизма экономической оценки рецепции «зеленой» энергетической практики пограничных регионов позволит руководству предприятий и организаций выявить возможности оптимизации показателей энергоемкости технологических процессов, экологической безопасности территорий, повысить качество жизни населения и сохранить ресурсы для будущих поколений.

Литература

1. Арбаев К. А., Арбаев Т. К., Калдыббаев Б. К. Перспективы использования альтернативных источников энергии в Кыргызстане // Вестник Ошского государственного университета. - 2018. - № 4. - С. 3-6.

2. Варфоломеев С. Д., Шевалеевский О. И. Биотопливо и фотоэлектричество. 2010. - Российские перспективы TheChemicalJournal. - No. 10. - C. 38-41.

3. Гавенко Е. А. Проблемы и перспективы использования альтернативных источников энергии в мировой экономике // Современная мировая экономика: проблемы и перспективы в эпоху развития цүифровых технологий и биотехнологии // Сборник статей Москва, 2019. - С. 208-209.

4. Гасанов О. М., Гусейнов Дж. И., Адгезалова Х. А., Мамедова Р. Ф. Развитие альтернативных источников энергии как путь сохранения экологии в Азербайджане // Альтернативная и интеллектуальная энергетика: Материаль Международной научно-практической конференции. - 2018. - С. $186-187$.

5. Гиниятуллина Э. И. Использование альтернативных источников энергии на производственных предприятиях // Поволжский научный вестник. - 2018. - № 1. - С. 12-17.

6. Косолапова Э. В. Альтернативные источники энергии как залог стабильного развития региона // Международный научный сельскохозяйственный журнал. - 2018. - T. 1. - № 3. - С. 15-20.

7. Кузнецова Д. И., Черникова О. П. Возможности использования гелиоэнергетики в Кемеровской области. Энергетика, информатика, инноваџии - 2016: Международная научно-техническая конференция: 
НИУ «МЭИ», 2016. - С. 130-134.

8. Международный год устойчивой энергетики для всех. [Электронный ресурс]. Режим доступа: https://www.un.org/ru/events/sustainableenergyforall/background.shtml, свободный. - Загл. с экрана.

9. Мирзаев С., Файзулина У., Хайруллаева С. К вопросу использования альтернативных источников энергии в Узбекистане // Молодежь и XXI век - 2019: материаль IX Международной молодежной научной конференции. - Курск, 2019. - С. 225-229.

10. Рудченко Г. А., Рудченко Ю. А. Применение альтернативных источников энергии в бытовом секторе Республики Беларусь // Экономика. Бизнес. Финансы. - 2017. - № 12. - С. 27-31.

11. Фирсова В. А., Черникова О. П. Оиенка эффективности инвестирования в модульную котельную на биотопливе // Актуальные проблемы экономики и управления в ХХІ веке: сборник научных статей. Новокузнеик, 2019. - Ч. 2. - С. 217-220.

12. Фирсова В. А., Черникова О. П. Оченка эффективности использования ветроэлектростанции // Актуальные проблемы экономики и управления в XXI веке: сборник научных статей. - Новокузнецк, 2019. - 4. 2. - C. 231-235.

13. Черникова О. П. Формирование экологической отчетности угледобываютих предприятий / О. П. Черникова, П. П. Баранов // Горный журнал. - 2018. - № 3. - С. 82-85.

14. Чумак Е. Ю. Развитие альтернативных источников энергии в США, Китае и Европе // Экономические исследования молодых ученых. Научное обозрение. - Москва, 2018. - С. 102-106.

15. Шевиова С. В., Жолудь Д. С. Анализ зарубежного опыта использования альтернативных видов энергии // Энергосбережение. Энергетика. Энергоаудит. - 2010. - № 6 (76). - C. 49-53.

16. Akhtar Hussain, Syed Muhammad Arif, Muhammad Aslam Emerging renewable and sustainable energy technologies: State of the ar Renewable and Sustainable Energy Reviews. Vol. 71, 2017. P. 12-28. [An electronic resource]. Access mode: https://unchronicle.un.org/article/sustainable-urban-energy-future/, https:// www.researchgate.net/

publica-

tion/251712497_Improvement_of_China_Energy_Label_System_to_Promote_Sustainable_Energy_Consumpti on, free. Heading from the screen.

17. Liuyang Zhan, Meiting Ju, Jinpeng Liu. 2011. Improvement of China Energy Label System to Promote Sustainable Energy Consumption Energy Procedia 5. P. 2308-2315.

18. Phillips L. and Smith P. Sustainable Urban Energy Is the Future UN Chronicle Vol. LII. No. 3. 2015. [An electronic resource]. Access mode: https://unchronicle.un.org/article/sustainable-urban-energy-future/, free. Heading from the screen.

19. Phillips L. and Smith P. Sustainable Urban Energy Is the Future UN Chronicle. Vol. LII. No. 3. 2015. Available at:

20. Pibin Guo, Juan Kong, Yanshan Guo, Xiuli Liu Identifying the influencing factors of the sustainable energy transitions in China Journal of Cleaner Production Vol. 215. 201. P. 757-766.

21. Rosemary N. Wojuola, Busisiwe P. Alant Sustainable development and energy education in Nigeria Renewable Energy Vol.139. 2019. P. 1366-1374.

22. Sustainable Energy // UN Chronicle Vol. LII. No. 3. 2015. [An electronic resource]. Access mode: https:// unchronicle.un.org/issue/sustainable-energy, free. Heading from the screen.

\section{References:}

1. Arbaev K. A., Arbaev T. K., Kaldybaev B. K. Perspektivy ispol'zovaniya al'ternativnyh istochnikov energii v Kyrgyzstane // Vestnik Oshskogo gosudarstvennogo universiteta. - 2018. - № 4. - S. 3-6.

2. Varfolomeev S. D., SHevaleevskij O. I. Biotoplivo $i$ fotoelektrichestvo. 2010. - Rossijskie perspektivy TheChemicalJournal. - No. 10. - S. 38-41.

3. Gavenko E. A. Problemy $i$ perspektivy ispol'zovaniya al'ternativnyh istochnikov energii $v$ mirovoj ekonomike // Sovremennaya mirovaya ekonomika: problemy $i$ perspektivy $v$ epohu razvitiya cifrovyh tekhnologij i biotekhnologii // Sbornik statej Moskva, 2019. - S. 208-209.

4. Gasanov O. M., Gusejnov Dzh. I., Adgezalova H. A., Mamedova R. F. Razvitie al'ternativnyh istochnikov energii kak put' sohraneniya ekologii v Azerbajdzhane // Al'ternativnaya i intellektual'naya energetika: Materialy Mezhdunarodnoj nauchno-prakticheskoj konferencii. - 2018. - S. 186-187.

5. Giniyatullina E. I. Ispol'zovanie al'ternativnyh istochnikov energii na proizvodstvennyh predpriyatiyah // Povolzhskij nauchnyj vestnik. - 2018. - № 1. - S. 12-17.

6. Kosolapova E. V. Al'ternativnye istochniki energii kak zalog stabil'nogo razvitiya regiona // Mezhdunarodnyj nauchnyj sel'skohozyajstvennyj zhurnal. - 2018. - T. 1. - № 3. - S. 15-20.

7. Kuznecova D. I., CHernikova O. P. Vozmozhnosti ispol'zovaniya gelioenergetiki v Kemerovskoj oblasti. Energetika, informatika, innovacii - 2016: Mezhdunarodnaya nauchno-tekhnicheskaya konferenciya: NIU «MEI», 2016. - S. 130-134.

8. Mezhdunarodnyj god ustojchivoj energetiki dlya vsekh. [Elektronnyj resurs]. Rezhim dostupa: https:// www.un.org/ru/events/sustainableenergyforall/background.shtml, svobodnyj. - Zagl. s ekrana.

9. Mirzaev S., Fajzulina U., Hajrullaeva S. K voprosu ispol'zovaniya al'ternativnyh istochnikov energii $v$ Uzbekistane // Molodezh' i XXI vek - 2019: materialy IX Mezhdunarodnoj molodezhnoj nauchnoj konferencii. Kursk, 2019. - S. 225-229.

10. Rudchenko G. A., Rudchenko YU. A. Primenenie al'ternativnyh istochnikov energii v bytovom sektore Respubliki Belarus' // Ekonomika. Biznes. Finansy. - 2017. - № 12. - S. 27-31.

11. Firsova V. A., CHernikova O. P. Ocenka effektivnosti investirovaniya v modul'nuyu kotel'nuyu na biotoplive // Aktual'nye problemy ekonomiki i upravleniya v XXI veke: sbornik nauchnyh statej. - Novokuzneck, 2019. - CH. 2. - S. 217-220. 
12. Firsova V. A., CHernikova O. P. Ocenka effektivnosti ispol'zovaniya vetroelektrostancii // Aktual'nye problemy ekonomiki i upravleniya v XXI veke: sbornik nauchnyh statej. - Novokuzneck, 2019. - CH. 2. - S. 231235.

13. CHernikova O. P. Formirovanie ekologicheskoj otchetnosti ugledobyvayushchih predpriyatij / O. P. CHernikova, P. P. Baranov // Gornyj zhurnal. - 2018. - № 3. - S. 82-85.

14. CHumak E. YU. Razvitie al'ternativnyh istochnikov energii v SSHA, Kitae i Evrope // Ekonomicheskie issledovaniya molodyh uchenyh. Nauchnoe obozrenie. - Moskva, 2018. - S. 102-106.

15. SHevcova S. V., ZHolud' D. S. Analiz zarubezhnogo opyta ispol'zovaniya al'ternativnyh vidov energii // Energosberezhenie. Energetika. Energoaudit. - 2010. - № 6 (76). - S. 49-53.

16. Akhtar Hussain, Syed Muhammad Arif, Muhammad Aslam Emerging renewable and sustainable energy technologies: State of the ar Renewable and Sustainable Energy Reviews. Vol. 71, 2017. P. 12-28. [An electronic resource]. Access mode: https://unchronicle.un.org/article/sustainable-urban-energy-future/, https:// www.researchgate.net/ publica-

tion/251712497 Improvement of China_Energy_Label System to Promote Sustainable Energy Consumpti on, free. Heading from the screen.

17. Liuyang Zhan, Meiting Ju, Jinpeng Liu. 2011. Improvement of China Energy Label System to Promote Sustainable Energy Consumption Energy Procedia 5. P. 2308-2315.

18. Phillips L. and Smith P. Sustainable Urban Energy Is the Future UN Chronicle Vol. LII. No. 3. 2015. [An electronic resource]. Access mode: https://unchronicle.un.org/article/sustainable-urban-energy-future/, free. Heading from the screen.

19. Phillips L. and Smith P. Sustainable Urban Energy Is the Future UN Chronicle. Vol. LII. No. 3. 2015. Available at:

20. Pibin Guo, Juan Kong, Yanshan Guo, Xiuli Liu Identifying the influencing factors of the sustainable energy transitions in China Journal of Cleaner Production Vol. 215. 201. P. 757-766.

21. Rosemary N. Wojuola, Busisiwe P. Alant Sustainable development and energy education in Nigeria Renewable Energy Vol.139. 2019. P. 1366-1374.

22. Sustainable Energy // UN Chronicle Vol. LII. No. 3. 2015. [An electronic resource]. Access mode: https:// unchronicle.un.org/issue/sustainable-energy, free. Heading from the screen. 